\title{
BMJ Open Uveitis as a potential predictor of acute myocardial infarction in patients with Behcet's disease: a population-based cohort study
}

\author{
Yu-Yen Chen (D) , 1,2,3,4 Yun-Ju Lai (1) , 2,5,6 Yung-Feng Yen (1) ,7,8,9 \\ Hsin-Hua Chen (D) ,2,3,10,11,12,13 Pesus Chou ${ }^{2,4}$
}

To cite: Chen Y-Y, Lai Y-J, Yen Y$\mathrm{F}$, et al. Uveitis as a potential predictor of acute myocardial infarction in patients with Behcet's disease: a populationbased cohort study. BMJ Open 2021;11:e042201. doi:10.1136/ bmjopen-2020-042201

- Prepublication history for this paper is available online. To view these files, please visit the journal online (http://dx.doi. org/10.1136/bmjopen-2020 042201).

Received 28 June 2020 Revised 14 December 2020 Accepted 29 December 2020

Check for updates

(c) Author(s) (or their employer(s)) 2021. Re-use permitted under CC BY-NC. No commercial re-use. See rights and permissions. Published by BMJ.

For numbered affiliations see end of article.

Correspondence to

Dr Yu-Yen Chen

yuyenchen.phd@gmail.com

\section{ABSTRACT}

Objectives To investigate whether uveitis is a predictor of acute myocardial infarction (AMI) among patients with Behcet's disease (BD).

Design Retrospective cohort study.

Setting Patients with BD were retrieved from the whole population of the Taiwan National Health Insurance

Research Database from 1 January 2001 to 31 December 2013.

Participants Among the 6508 patients with BD, 2517

$(38.7 \%)$ were in the uveitis group and 3991 were in the non-uveitis group.

Primary and secondary outcome measures KaplanMeier curves were generated to compare the cumulative hazard of AMI in the uveitis and non-uveitis groups. Multivariate Cox regression analysis was used to estimate the adjusted $\mathrm{HRs}$ and $95 \% \mathrm{Cl}$ of $\mathrm{AMI}$, and was adjusted for age, gender, systemic comorbidities (eg, hypertension, diabetes, hyperlipidaemia, smoking) and clinical manifestation of BD (eg, oral ulcers, genital ulcers, skin lesions, arthritis and gastrointestinal involvement). Results The mean age of the BD cohort was $38.1 \pm 15.1$ years. Compared with non-uveitis patients, uveitis patients were significantly younger and male predominant. There was no significant difference between the two groups for most proportions of systemic comorbidities and clinical manifestations. The Kaplan-Meier method with the logrank test showed that the uveitis group had a significantly higher cumulative hazard for patients with AMI compared with the non-uveitis group $(p<0.0001)$. In the multivariable Cox regression after adjustment for confounding factors, patients with uveitis had a significantly higher risk of AMI (adjusted HR 1.87; 95\% $\mathrm{Cl} 1.52$ to 2.29). Other significant risk factors for AMI were age, hypertension, smoking, and skin lesions.

Conclusions Statistical analyses from the nationwide database demonstrated that uveitis is a potential predictor of $A M I$ in patients with $B D$.

\section{INTRODUCTION}

Behcet's disease (BD) is a systemic inflammatory disease characterised by recurrent oral ulcers, genital ulcers, uveitis and skin lesions. Arthritis, gastrointestinal ulcers and vascular involvement might also be present.
Strengths and limitations of this study

- The study used the comprehensive, entire national population database, with a 13-year study period to investigate the predictors of acute myocardial infarction (AMI) in patients with Behcet's disease.

- Possible confounders were adjusted using Cox regression, to elucidate how uveitis and AMI are correlated in patients with Behcet's disease.

- The retrospective cohort design allowed us to explore associations, but not causality.

BD vasculitis occurs in all sizes of arteries and veins, ${ }^{1-3}$ primarily manifesting in venous thrombosis, but tend to be more devastating in arterial lesions, such as aneurysms, thrombosis, occlusion and stenosis. ${ }^{4}$ Although coronary artery involvement is extremely rare in $\mathrm{BD},{ }^{67}$ it is among the most life-threatening complications when acute myocardial infarction (AMI) occurs. Population-based studies have demonstrated that there is a greater risk of AMI or ischaemic heart disease in patients with BD compared with controls. ${ }^{78}$ The underlying mechanism of AMI might attributed to systemic inflammation in BD leading to coronary arteritis. Disruption of the internal elastic lamina of the coronary artery might lead to the formation of aneurysms, potentially impairing blood flow, leading to the formation of thrombus and, consequently, AMI. ${ }^{9} 10$ In addition, injured vascular endothelia are prone to atherosclerosis and thrombosis, which are established precipitating factors of AMI. ${ }^{2} 1112$

Uveitis is an intraocular inflammation that is a major presentation in BD. Uveitis is diagnosed using non-invasive techniques, such as slit lamp examination and funduscopy. Because of the elevated levels of cytokine in the aqueous humour of patients with uveitis, ${ }^{13}$ uveitis might indicate higher 
severity of systemic inflammation in BD. Furthermore, more inflammation may induce more vascular endothelial injuries, cascading towards AMI. Therefore, uveitis might be a predictor of AMI in patients with BD; however, this hypothesis has not been tested.

Due to the serious consequences of AMI, it is important to understand how $\mathrm{BD}$ and $\mathrm{AMI}$ are associated, including predictors for AMI in patients with $\mathrm{BD}$. However, AMI is rare in $\mathrm{BD}$; consequently, previous studies were limited to case reports or case series. ${ }^{14-20}$ Few studies exist with large enough sample-sizes to define clear predictors for AMI in BD. ${ }^{12}{ }^{21}$ Furthermore, the clinical signs of BD vary in relation to ethnicity and geography.

Therefore, here, we aimed to fill this knowledge gap on the characteristics of $\mathrm{BD}$ and contribution to the development of AMI in people in Taiwan. We used the National Health Insurance Research Database (NHIRD) of the entire population of 23 million people in Taiwan. This approach resulted in our having a larger number numbers of patients with BD compared with previous studies. Specifically, we aimed to understand the clinical manifestations of BD in Taiwan, and elucidate whether uveitis or other features could be used as predictors of $\mathrm{AMI}$ in BD.

\section{MATERIALS AND METHODS \\ Data source}

Taiwan NHIRD contains comprehensive medical records, including information on outpatients, inpatients, emergency services, prescriptions, procedures, surgeries and diagnoses, which are registered using the International Classification of Diseases, Ninth Revision, Clinical Modification (ICD-9-CM) codes. The identity of all patients included in the database was encrypted before data were released for research purposes; thus, the requirement for informed consent was waived by the institutional review board.

\section{Patient involvement}

Patients were not directly involved in the design of this study.

\section{Study design and sample}

We conducted a retrospective cohort study from 1 January 2001 to 31 December 2013. First, we selected patients presenting with BD (ICD-9-CM code 136.1) during the study period. During the study period, the diagnostic criteria in Taiwan fulfilled 1990 International Study Group BD criteria. ${ }^{22}$ Those diagnosed with BD before the end of 2000 were excluded, to ensure that all enrolled patients had new onset of BD. The date of first BD diagnosis was defined as the index date. Second, patients in the study cohort were monitored to identify the onset of AMI. During the study period in Taiwan, AMI was diagnosed according to the WHO category B definition of MI. ${ }^{23}$ Those diagnosed with AMI before the index date were excluded, to ensure that all included AMI cases were new onset after BD was diagnosed. The study cohort was followed until AMI occurred, patients were dropped from the database, or the end of 2013 was reached, whichever came first.

\section{Variable definitions}

As this study examined how uveitis impacts the risk of AMI, the main independent variable of interest was uveitis. Previous studies suggested that risk factors of AMI include age, gender and certain comorbidities, such as hypertension, diabetes, hyperlipidaemia, smoking (or chronic obstructive pulmonary disease). ${ }^{24}$ These factors were considered to be confounders, and were adjusted for in our statistical analyses. We also derived the clinical characteristics of BD found in previous study, ${ }^{25}$ from the diagnoses of oral ulcers, genital ulcers, skin lesions, arthritis, as well as gastrointestinal, vascular and neurological involvement to investigate whether any of these factors are predictors of AMI.

\section{Statistical analysis}

All enrolled patients with BD were separated into a uveitis group or non-uveitis group, based on whether they had uveitis. After investigating the two groups descriptively by age, gender, comorbidities (eg, hypertension, diabetes, hyperlipidaemia, smoking) and clinical manifestations of BD (eg, oral ulcers, genital ulcers, skin lesions, arthritis and gastrointestinal involvement), the group differences were analysed by the two-sample t-test (for continuous variables) and $\chi^{2}$ test (for categorical variables). Survival analysis was performed using the Kaplan-Meier method, with the log-rank test being used to describe and compare the cumulative hazard curves of AMI. A Cox proportional hazard model was used to estimate the HR for the occurrence of AMI based on each variable in the univariate and multivariate analyses. Variables included in the regression analysis were age, gender, comorbidities and clinical features of BD. All statistical analyses were performed using SAS statistical package, V.9.4 (SAS Institute).

\section{RESULTS}

Demographic and clinical characteristics of the study sample

This study included 6508 subjects with BD (table 1). Out of the study cohort, 2517 (38.7\%) patients had uveitis (uveitis group) and 3991 (61.3\%) had no uveitis (nonuveitis group). The mean age of the total BD cohort was 38.1 years (SD 15.1 years), with almost $55 \%$ of patients being less than 40 years old. Females represented $56.4 \%$ of the cohort. The prevalence of both hypertension and hyperlipidaemia was almost $20 \%$. Fewer patients $(9.4 \%)$ had diabetes. Those who smoked accounted for almost $6.0 \%$ of the study cohort. The most common clinical features of BD were oral ulcers $(99.0 \%)$ and genital ulcers $(95.3 \%)$, followed by skin lesion $(56.0 \%)$, gastrointestinal involvement (30.1\%), and arthritis (26.9\%). As far as vascular manifestations were concerned, venous thrombosis, arterial thrombosis and arterial aneurysm 
Table 1 Characteristics of the study subjects

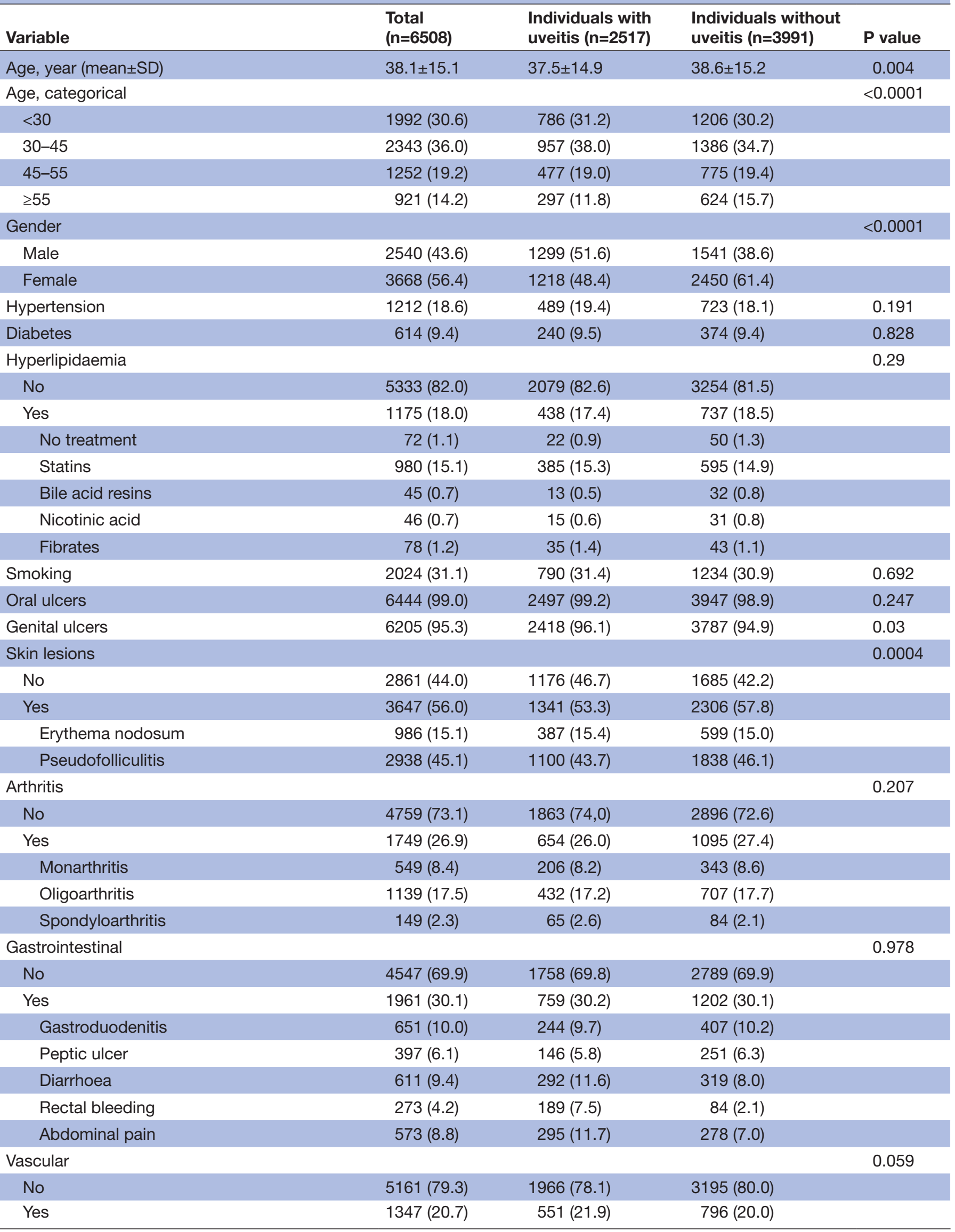




\begin{tabular}{|c|c|c|c|c|}
\hline Variable & $\begin{array}{l}\text { Total } \\
(n=6508)\end{array}$ & $\begin{array}{l}\text { Individuals with } \\
\text { uveitis }(n=2517)\end{array}$ & $\begin{array}{l}\text { Individuals without } \\
\text { uveitis }(n=3991)\end{array}$ & $P$ value \\
\hline Venous thrombosis & $1320(20.3)$ & $538(21.4)$ & 782 (19.6) & \\
\hline Arterial aneurysm & $534(8.2)$ & $211(8.4)$ & $323(8.1)$ & \\
\hline Arterial thrombosis & $677(10.4)$ & $279(11.1)$ & $398(10.0)$ & \\
\hline Neurological & & & & 0.218 \\
\hline No & $5825(89.5)$ & 2238 (88.9) & 3587 (89.9) & \\
\hline Yes & $683(10.5)$ & $279(11.1)$ & $404(10.1)$ & \\
\hline Central & $25(0.4)$ & $15(0.6)$ & $10(0.3)$ & \\
\hline Peripheral & $237(3.6)$ & $98(3.9)$ & 139 (3.5) & \\
\hline Headache & $479(7.4)$ & $194(7.7)$ & $285(7.1)$ & \\
\hline Follow-up period, year (mean $\pm S D$ ) & $6.1 \pm 3.7$ & $6.2 \pm 3.7$ & $6.0 \pm 3.6$ & 0.08 \\
\hline AMI during the follow-up period & $382(5.9)$ & $210(8.3)$ & $172(4.3)$ & $<0.0001$ \\
\hline
\end{tabular}

Data are presented as mean \pm SD or $\mathrm{n}(\%)$.

AMI, acute myocardial infarction; SD, standard deviation.

constituted $20.3 \%, 10.4 \%$, and $8.2 \%$ of all vascular lesions, respectively. When the uveitis and non-uveitis groups were compared, the age of patients in the uveitis group was significantly younger, with a predominance of males. Prevalence of comorbidities such as hypertension, diabetes, hyperlipidaemia and smoking were similarly distributed between the uveitis group and the non-uveitis group. Most of the clinical features of BD also presented at similar frequencies in both groups. During the follow-up period, the cumulative incidence of AMI was significantly higher in the uveitis group $(8.3 \%)$ compared with the non-uveitis group $(4.3 \%)$.

\section{Cumulative hazard curves using the Kaplan-Meier method}

Figure 1 shows the cumulative hazard curves for AMI for patients with $\mathrm{BD}$ in the uveitis and non-uveitis groups.

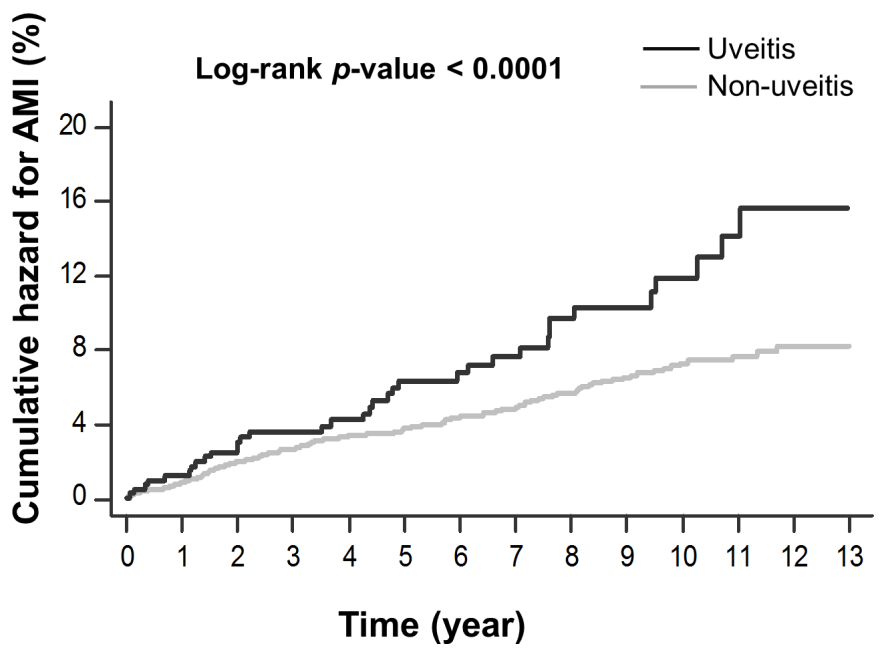

Figure 1 Kaplan-Meier curves for acute myocardial infarction (AMI) in patients with Behcet's disease, corresponding to the uveitis and non-uveitis groups. Black line represents the uveitis group; grey line represents the nonuveitis group.
A log-rank test showed a statistically significant higher hazard in the uveitis group compared with the non-uveitis group $(\mathrm{p}<0.0001)$.

\section{Univariate and multivariate analyses using the Cox regression model}

The unadjusted HR for AMI was 1.70 times greater in the uveitis group compared with the non-uveitis group (95\% CI 1.39 to 2.08) (table 2). After adjusting for covariates, the significantly greater HR for AMI in the uveitis group remained (adjusted HR 1.87; 95\% CI 1.52 to 2.29). Age was a significant risk factor for AMI in both univariate and multivariate analyses. The adjusted HR for AMI in patients over 55 years old was 6.97 when compared with patients younger than 30 years old. Males were significantly more likely to develop AMI compared with females in the univariate analysis; however, this difference was not significant after adjusting confounders (adjusted HR 1.05; 95\% CI 0.89 to 1.29 ). In the univariate analysis, patients with hypertension, diabetes, hyperlipidaemia and smoking had a significantly higher risk of developing AMI (all $\mathrm{p}<0.0001$ ); however, only hypertension and smoking remained significant after adjusting the covariates (adjusted HR 2.36 with 95\% CI 1.85 to 2.99 and adjusted HR 1.55 with $95 \%$ CI 1.35 to 1.81 , respectively). Skin lesions and arthritis were clinical characteristics of BD that were significantly associated with an increased the risk of AMI in the univariate analysis. Skin lesions remained significant in the multivariate analysis (adjusted HR 1.51 ; $95 \%$ CI 1.17 to 1.93$)$.

\section{Characteristics of the patients with AMI}

Table 3 focuses on patients with AMI events. Duration between $\mathrm{BD}$ and $\mathrm{AMI}$ were calculated among the uveitis and non-uveitis groups (mean \pm SD: $4.5 \pm 3.0$ years

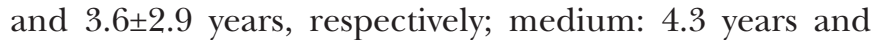
2.6 years, respectively). Left anterior descending artery 
Table 2 Analyses of risk factors for AMI in patients with BD with and without uveitis

\begin{tabular}{|c|c|c|c|c|}
\hline \multirow[b]{2}{*}{ Predictive variables } & \multicolumn{2}{|l|}{ Univariate analysis } & \multicolumn{2}{|l|}{ Multivariate analysis } \\
\hline & Unadjusted HR $(95 \% \mathrm{Cl})$ & $P$ value & Adjusted HR (95\% Cl) & $P$ value \\
\hline Uveitis (yes vs no) & 1.70 (1.39 to 2.08$)$ & $<0.0001$ & 1.87 (1.52 to 2.29$)$ & $<0.0001$ \\
\hline$<30$ & Reference & & Reference & \\
\hline $30-45$ & 4.37 (2.82 to 6.75$)$ & $<0.0001$ & 3.28 (2.11 to 5.11$)$ & $<0.0001$ \\
\hline Gender (male vs female) & 1.12 (0.92 to 1.37$)$ & 0.263 & 1.05 (0.85 to 1.29$)$ & 0.651 \\
\hline Hypertension & 4.57 (3.74 to 5.59$)$ & $<0.0001$ & 2.36 (1.85 to 2.99$)$ & $<0.0001$ \\
\hline Diabetes & 2.18 (1.69 to 2.81$)$ & $<0.0001$ & $1.02(0.93$ to 1.17$)$ & 0.471 \\
\hline Hyperlipidaemia & 2.71 (2.21 to 3.33$)$ & $<0.0001$ & 1.15 (0.91 to 1.45$)$ & 0.257 \\
\hline Smoking & 3.29 (2.74 to 3.89$)$ & $<0.0001$ & 1.55 (1.35 to 1.81$)$ & 0.0001 \\
\hline Oral ulcers & $1.84(0.46$ to 7.37$)$ & 0.391 & 1.49 (0.37 to 5.98$)$ & 0.576 \\
\hline Genital ulcers & 1.07 (0.65 to 1.77$)$ & 0.784 & 1.01 (0.61 to 1.69$)$ & 0.953 \\
\hline Skin lesions & 2.40 (1.89 to 3.03$)$ & $<0.0001$ & 1.51 (1.17 to 1.93$)$ & 0.001 \\
\hline Arthritis & 1.54 (1.25 to 1.89$)$ & $<0.0001$ & 1.11 (0.90 to 1.38$)$ & 0.323 \\
\hline Gastrointestinal involvement & 1.05 (0.88 to 1.26$)$ & 0.574 & $1.06(0.89$ to 1.10$)$ & 0.844 \\
\hline
\end{tabular}

In the multivariate analysis, all other variables in the table were included for adjustment.

$\mathrm{AMI}$, acute myocardial infarction; BD, Behcet's disease; $\mathrm{Cl}$, confidence interval; HR, hazard ratio.

(LAD) is the most frequently involved coronary artery, followed by right coronary artery (RCA) and left circumflex artery (LCx). Besides, our study found the the most common aetiology of AMI except stenosis was thrombosis. Types of skin lesions, arthritis and gastrointestinal manifestations were also presented in table 3 . The most frequent signs among the three categories were pseudofolliculitis, oligoarthritis and gastroduodenitis/diarrhoea. Furthermore, patients with AMI in the uveitis group had a significantly higher prevalence of skin lesions than the patients with AMI in the non-uveitis group $(65.2 \%$ vs $44.8 \%, \mathrm{p}<0.0001)$.

\section{DISCUSSION}

We conducted a 13-year cohort study on populationbased data from the Taiwan NHIRD. Our study included 6508 patients with new onset of BD. Out of these patients, $2517(38.7 \%)$ had uveitis and 3991 (61.3\%) did not have uveitis. Compared with patients without uveitis, those with uveitis had a significantly higher risk $(\mathrm{HR}=1.87)$ of developing AMI after adjusting for age, gender, comorbidities and the clinical characteristics of BD.

$\mathrm{BD}$ is most common in countries along the ancient Silk Road, extending from the Mediterranean Sea to east Asia. For example, the incidence of BD per 100000 people is highest in Turkey (21), but is much lower in Korea (4.0), Taiwan (2.4), the USA (0.1-0.3) and Western Europe $(0.2-1) .{ }^{26-31}$ Also, the male-to-female ratio in BD varies across countries. $\mathrm{BD}$ is more prevalent in males in Silk Route areas, is equally distributed between the genders in the USA and western Europe, and is more prevalent in females in Japan and South Korea. ${ }^{72-35}$ In the current study of patients with BD in Taiwan, females constituted $56.4 \%$ of patients. This percentage reflects that of previous studies in Taiwan. ${ }^{29} 36$ Of interest, our study showed that the onset of $\mathrm{BD}$ was more prevalent in younger people (mean 38.1 years), with more than half of patients being under 40 years old. These results were consistent with previous studies showing that BD typically affects people of 20-40 years old. The current study also showed that the prevalence of hypertension, diabetes and hyperlipidaemia in patients with BD uveitis was similar to a previous report using the sampled NHIRD in Taiwan. ${ }^{36}$

Table 1 shows that nearly $40 \%$ of patients with BD had uveitis. The percentage of patients with BD tends to vary with region (eg, $18 \%-65 \%$ in Asia-Pacific countries). ${ }^{2937-39}$ We also evaluated how clinical expression (systemic and ocular) differed between uveitis and non-uveitis groups, which was novel compared with previous studies. We found that most of the baseline characteristics of the two groups were not significantly different, implying their comparability. Thus, the significant increase in the cumulative incidence and cumulative hazard of AMI in uveitis patients (table 1, figure 1) was less likely to be attributed to inherent, baseline differences in systemic diseases. 
Table 3 Characteristics of patients with AMI in the study cohort

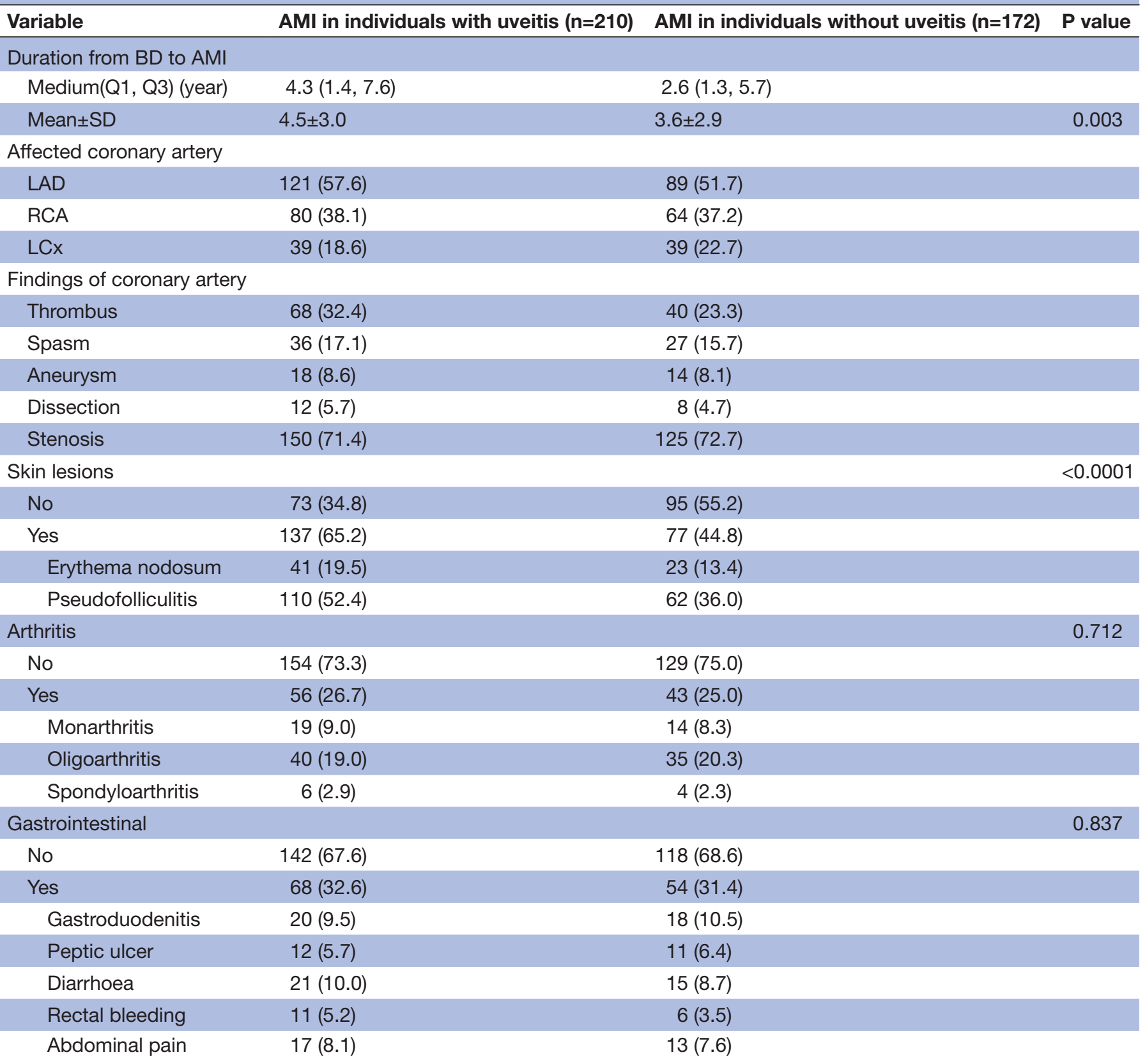

Data are presented as $\mathrm{n}(\%)$.

AMI, acute myocardial infarction; BD, Behcet's disease; LAD, left anterior descending artery; LCx, left circumflex artery; RCA, right coronary artery; SD, standard deviation.

To reduce confounding effects, we included systemic comorbidities for adjustment in the Cox regression analysis. Age, hypertension and smoking are traditional risk factors for AMI among general population, ${ }^{24}$ and, as expected, represented significant risk factors for AMI among patients with BD. Previous cross-sectional and case-control studies on the association between BD and AMI showed very similar results. Pandey et al found that hypertension and hyperlipidaemia were independent predictors of coronary artery disease in patients with $\mathrm{BD}$, based on the Nationwide Inpatient Sample database of the USA. ${ }^{12}$ Kechida et al reported male gender and skin lesions as independent risk factors for cardiac and vascular involvement in a retrospective study of patients with BD in a tertiary hospital in north Africa. ${ }^{40}$ Our study identified skin lesions as a predictive factor of AMI in BD when including clinical manifestations for adjustment in the Cox regression.

The aetiologies of how skin lesions are associated with cardiovascular involvement in BD are not known. A previous case report described the pathology of the skin lesions (eg, erythema nodosum) as the infiltration of neutrons and lymphocytes in the dermis and around the blood vessels of the subcutaneous fat tissue. ${ }^{41}$ Thus, 
skin lesions might predict the occurrence of AMI because they represent an inflammatory reaction, leading to AMI. The same case report showed that AMI occurs during the exacerbations of skin lesions, suggesting that skin lesions and AMI are associated.

It is noteworthy that we investigate the clinical characteristics of patients with AMI in our study cohort. Similar to the result of Chen $e$ al's study, ${ }^{21}$ our study found the order of affected coronary artery was LAD, RCA and LCx (from the most frequent to the least frequent). Besides, thrombosis was the the most common aetiology of AMI except stenosis. The echocardiographic study conducted by Farouk et al revealed the presence of intracardiac thrombi in four out of five patients with BD with cardiac symptoms. ${ }^{42}$ And, intracardiac or intracoronary thrombosis might be an important cause of AMI in patients with $\mathrm{BD}$. The high prevalences of peripheral venous thrombosis $(20 \%)$ and arterial thrombosis ( $10 \%)$ presented in table 1 were compatible with Vural's study in Turkey. ${ }^{20}$ Furthermore, our study revealed a higher prevalence of coronary artery thrombosis in AMI among the uveitis group than in the non-uveitis group, implying tendency to thrombosis could also be related to the occurrence of AMI, especially in the uveitis group.

The reason why uveitis might indicate a higher risk of AMI is not fully understood. Previous studies have suggested that one of the etiologies of AMI is inflammation, which may induce the process of thrombosis or atherosclerosis. ${ }^{43-46}$ Canakinumab Anti-inflammatory Thrombosis Outcomes Study (CANTOS) evaluated whether inhibition of interleukin-1B (IL-1B), a proinflammatory cytokine could reduce the rate of major adverse cardiovascular event (MACE). Canakinumab, a human monoclonal antibody that selectively neutralises IL-1B, with modulation of IL-6, was found to be associated with reduced MACE rate. ${ }^{47}$ CANTOS provides evidences that inflammation may cause AMI. Uveitis in BD includes anterior uveitis, posterior uveitis and retinal vasculitis. Molecular studies have demonstrated that intraocular $\mathrm{T}$ cells, cytotoxic cells and dendritic cells contribute to inflammation, and augment the immune reaction of Behcet's uveitis. ${ }^{48-50}$ In addition, higher expressions of maturation markers in dendritic cells might represent the activity of disease in Behcet's uveitis. ${ }^{51}$ Therefore, uveitis might reflect higher inflammatory activity in BD. Systemic inflammation in BD leads to vasculitis, with greater intima-media thickness and lower arterial distensibility, predisposing patients to vascular stenosis. ${ }^{52}$ In addition, endothelial dysfunction causes more von Willebrand factor to be released, decreasing fibrinolysis. ${ }^{53-55}$ All these factors precipitate thrombosis, which causes AMI if it occurs in a coronary artery. Thus, the risk of AMI increases when inflammation activity is higher. Because uveitis in BD reflects a more severe inflammatory reaction, uveitis might be a predictor of $\mathrm{AMI}$ in $\mathrm{BD}$.

The current study was limited because it was only based on statistical analyses from the database, and lacked laboratory evidences such as $\mathrm{C}$ reactive protein, erythrocyte sedimentation rate, haemoglobin and human leucocyte antigen to elucidate the underlying mechanisms. Besides, the ECG findings were not recorded in our database. Therefore, we did not know the proportion of ST-segment elevation in our patients with AMI. Another limitation was that some factors (such as dietary habits and physical activity) that impact AMI were not included in our study because the database lacked this information. Furthermore, we were only able to derive association, not causality, because of our retrospective study design. Thus, further laboratory and clinical studies with a prospective design, as well as detailed information on lifestyle (eg, diet and exercise) are required to elucidate the predictors, risk factors, and underlying mechanisms of AMI in patients with BD.

One strength of our study was that we used a comprehensive, nationwide database spanning 13 years. The large case numbers gave the statistical analyses higher power. Another strength was that we included systemic diseases and clinical manifestations of BD in the cox regression to identify significant factors associated with AMI. In the healthcare system of Taiwan, the National Health Administration checks charts, and ensures that patients receive a standard protocol of examinations to confirm diagnoses. Therefore, the diagnoses were accurate and verified. Our study showed that uveitis might be a predictor of AMI in patients with BD. Moreover, age, hypertension, smoking and skin lesions were also significantly associated with a higher risk of AMI. These findings were obtained after adjusting for confounders and, thus, are considered reliable and convincing.

\section{CONCLUSIONS}

Using the entire NHIRD population in Taiwan over 13 years, we demonstrated that uveitis is associated with a significantly higher risk of AMI in patients with BD. Doctors should be aware of the potential threats of AMI in patients with $\mathrm{BD}$. Close monitoring of the cardiovascular system by cardiologists is imperative, especially for patients with uveitis or other risk factors, to detect AMI risk early and to manage it promptly.

\section{Author affiliations}

${ }^{1}$ Department of Ophthalmology, Taichung Veterans General Hospital, Taichung, Taiwan

${ }^{2}$ School of Medicine, National Yang-Ming University, Taipei, Taiwan

${ }^{3}$ School of Medicine, Chung Shan Medical University, Taichung, Taiwan

${ }^{4}$ Community Medicine Research Center and Institute of Public Health, National Yang-Ming University, Taipei, Taiwan

${ }^{5}$ Division of Endocrinology and Metabolism, Department of Internal Medicine, Puli Branch of Taichung Veterans General Hospital, Nantou, Taiwan

${ }^{6}$ Department of Exercise Health Science, National Taiwan University of Sport, Taichung, Taiwan

${ }^{7}$ Section of Infectious Diseases, Taipei City Hospital, Yangming Branch, Taipei, Taiwan

${ }^{8}$ Institute of Public Health, National Yang-Ming University, Taipei, Taiwan

${ }^{9}$ Department of Health Care Management, National Taipei University of Nursing and Health Sciences, Taipei, Taiwan 
${ }^{10}$ Department of Medical Research, Taichung Veterans General Hospital, Taichung, Taiwan

${ }^{11}$ Division of Allergy, Immunology and Rheumatology, Department of Internal

Medicine, Taichung Veterans General Hospital, Taichung, Taiwan

${ }^{12}$ Institute of Biomedical Science and Rong-Hsing Research Center for Translational Medicine, Chung-Hsing University, Taichung, Taiwan

${ }^{13}$ Department of Industrial Engineering and Enterprise Information, Tunghai University, Taichung, Taiwan

Contributors Conceptualisation: Y-YC, Y-JL, Y-FY and PC. Formal analysis: Y-YC, Y-JL and PC. Investigation: Y-YC and Y-FY. Methodology: Y-YC, Y-JL, Y-FY and H-HC. Validation: $Y-Y C, P C$ and $H-H C$. Writing the original draft: $Y-Y C$.

Funding This work was supported by the Taichung Veterans General Hospital (grant number TCVGH-1096902B).

Competing interests None declared.

Patient consent for publication Not required.

Ethics approval This study was approved by the ethics committee of Yang-Ming University Hospital (2015A018).

Provenance and peer review Not commissioned; externally peer reviewed.

Data availability statement Data are available on reasonable request. Data are available from the National Health Insurance Research Database (NHIRD) published by Taiwan National Health Insurance (NHI) Bureau. The data used in this study cannot be made available in the manuscript, online supplemental files or in a public repository due to the Personal Information Protection Act executed by Taiwan's government, starting from 2012. Requests for data can be sent as a formal proposal to the NHIRD (http://nhird.nhri.org.tw) or by email to wt.gro.irhn@drihn.

Open access This is an open access article distributed in accordance with the Creative Commons Attribution Non Commercial (CC BY-NC 4.0) license, which permits others to distribute, remix, adapt, build upon this work non-commercially, and license their derivative works on different terms, provided the original work is properly cited, appropriate credit is given, any changes made indicated, and the use is non-commercial. See: http://creativecommons.org/licenses/by-nc/4.0/.

\section{ORCID iDs}

Yu-Yen Chen http://orcid.org/0000-0003-0891-3819

Yun-Ju Lai http://orcid.org/0000-0001-8252-2125

Yung-Feng Yen http://orcid.org/0000-0002-4469-4732

Hsin-Hua Chen http://orcid.org/0000-0002-7304-4587

\section{REFERENCES}

1 Sarica-Kucukoglu R, Akdag-Kose A, Kayaball M, et al. Vascular involvement in Behçet's disease: a retrospective analysis of 2319 cases. Int J Dermatol 2006;45:919-21.

2 Uyar B, Solak A, Genç B, et al. Evaluation of arterial stiffness in patients with Behçet's disease by using noninvasive radiological methods such as intima-media thickness of the carotid, Anklebrachial pressure index, coronary artery calcium scoring, and their relation to serum fetuin-A levels: a case-control study. Ann Dermatol 2015;27:702-8.

3 Kaya E, Saglam H, Ciftci I, et al. Evaluation of myocardial perfusion and function by gated SPECT in patients with Behçet's disease. Ann Nucl Med 2008;22:287-95.

4 Fei Y, Li X, Lin S, et al. Major vascular involvement in Behçet's disease: a retrospective study of 796 patients. Clin Rheumatol 2013;32:845-52.

5 Hatemi G, Seyahi E, Fresko I, et al. Behçet's syndrome: a critical digest of the 2014-2015 literature. Clin Exp Rheumatol 2015;33:S3-14.

6 Geri G, Wechsler B, Thi Huong DL. Spectrum of cardiac lesions in Behçet disease: a series of 52 patients and review of the literature. Medicine 2012;91:25-34.

7 Ahn HS, Lee D, Lee SY. Increased cardiovascular risk and allcause death in patients with Behçet disease: a Korean nationwide population-based dynamic cohort study 2020;47:903-8.

8 Yavne $\mathrm{Y}$, Tiosano S, Watad A, et al. Investigating the link between ischemic heart disease and Behcet's disease: a cross-sectional analysis. Int J Cardiol 2017;241:41-5.

9 Messaoud MB, Bouchahda N, Belfekih A, et al. A giant aneurysm of the left anterior descending coronary artery in the setting of Behcet's disease. Cardiovasc J Afr 2020;31:e1-3.
10 Pu L, Li R, Xie J, et al. A giant pseudoaneurysm of coronary artery in a young patient with Behçet's disease. Echocardiography 2017;34:1736-7.

11 Springer J, Villa-Forte A. Thrombosis in vasculitis. Curr Opin Rheumatol 2013;25:19-25.

12 Pandey A, Garg J, Krishnamoorthy P, et al. Predictors of coronary artery disease in patients with Behçet's disease. Cardiology 2014;129:203-6.

13 Valentincic NV, de Groot-Mijnes JDF, Kraut A, et al. Intraocular and serum cytokine profiles in patients with intermediate uveitis. Mol Vis 2011;17:2003-10.

14 Ayari J, Mourali MS, Farhati A, et al. Left main coronary artery thrombosis revealing angio-Behçet syndrome. Egypt $J$ Intern Med 2014;26:88-90.

15 Doğan A, Celik A, Doğan S, et al. Acute myocardial infarction due to a large coronary aneurysm in Behçet's disease. Turk Kardiyol Dern Ars 2011;39:737.

16 So H, Yip ML. Acute myocardial infarction and subclavian artery occlusion in a 41-year-old woman with Behçet's disease: coronary and large vessel arteritis. Singapore Med J 2014;55:e145-7.

17 Beyranvand M-R, Namazi M-H, Mohsenzadeh Y, et al. Acute myocardial infarction in a patient with Behçet's disease. Arch Iran Med 2009;12:313-6.

18 Guo Y, Tang L, Tang J, et al. Recurrent myocardial infarction due to coronary artery aneurysm in Behçet's syndrome: a case report. Eur Heart J Case Rep 2019;3:1-4.

19 Saad Shaukat MH, Toledo-Garcia A, Torosoff M. Recurrent myocardial infarction despite normal C-reactive protein in a patient with Behcet's disease and compound heterozygous methylenetetrahydrofolate reductase (MTHFR) mutations (C677T and A1298C). Cureus 2019;11:e5344

20 Vural U, Kizilay M, Aglar AA. Coronary involvement in Behçet's disease: what are its risks and prognosis? (rare cases and literature review). Braz J Cardiovasc Surg 2020;34:749-58.

21 Chen $\mathrm{H}$, Zhang Y, Li C, et al. Coronary involvement in patients with Behçet's disease. Clin Rheumatol 2019;38:2835-41.

22 International Study Group for Behcet's Disease. Criteria for diagnosis of Behçet's disease. International Study Group for Behçet's disease. Lancet 1990;335:1078-80.

23 Mendis S, Thygesen K, Kuulasmaa K, et al. World Health Organization definition of myocardial infarction: 2008-09 revision. Int $J$ Epidemiol 2011;40:139-46.

24 AHA, ACC, National Heart, Lung, and Blood Institute, et al. AHA/ ACC guidelines for secondary prevention for patients with coronary and other atherosclerotic vascular disease: 2006 update endorsed by the National heart, lung, and blood Institute. J Am Coll Cardiol 2006;47:2130-9.

25 Yazici H, Seyahi E, Hatemi G, et al. Behçet syndrome: a contemporary view. Nat Rev Rheumatol 2018;14:107-19.

26 Keino H, Okada AA. Behçet's disease: global epidemiology of an old silk road disease. Br J Ophthalmol 2007;91:1573-4.

27 Tüzün Y, Yurdakul S, Cem Mat M, et al. Epidemiology of Behçet's syndrome in turkey. Int J Dermatol 1996;35:618-20.

28 Lee YB, Lee SY, Choi JY, et al. Incidence, prevalence, and mortality of Adamantiades-Behçet's disease in Korea: a nationwide, population-based study (2006-2015). J Eur Acad Dermatol Venereol 2018;32:999-1003.

29 Lin Y-H, Tai T-Y, Pu C-Y, et al. Epidemiology of Behcet's disease in Taiwan: a population-based study. Ophthalmic Epidemiol 2018;25:323-9.

30 Calamia KT, Wilson FC, Icen M, et al. Epidemiology and clinical characteristics of Behcet's disease in the US: a population-based study. Arthritis Rheum 2009;61:600-4.

31 Zouboulis CC, Kötter I, Djawari D, et al. Epidemiological features of Adamantiades-Behçet's disease in Germany and in Europe. Yonsei Med J 1997;38:411-22.

32 Davatchi F, Shahram F, Chams-Davatchi C, et al. Behcet's disease: from East to West. Clin Rheumatol 2010;29:823-33.

33 Davatchi F, Chams-Davatchi C, Shams H, et al. Adult Behcet's disease in Iran: analysis of 6075 patients. Int $J$ Rheum Dis 2016;19:95-103.

34 Tugal-Tutkun I, Onal S, Altan-Yaycioglu R, et al. Uveitis in Behçet disease: an analysis of 880 patients. Am J Ophthalmol 2004;138:373-80.

35 Alpsoy E. Behçet's disease: a comprehensive review with a focus on epidemiology, etiology and clinical features, and management of mucocutaneous lesions. J Dermatol 2016;43:620-32.

$36 \mathrm{CY}$ W, HS Y, Chai CY. Increased ischemic stroke risk in patients with Behçet's disease: A nationwide population-based cohort study 2019;14:e0218652. 
37 Ideguchi H, Suda A, Takeno M, et al. Behçet disease: evolution of clinical manifestations. Medicine 2011;90:125-32.

38 Zhang Z, He F, Shi Y. Behcet's disease seen in China: analysis of 334 cases. Rheumatol Int 2013;33:645-8.

$39 \mathrm{Kim}$ DY, Choi MJ, Cho S, et al. Changing clinical expression of Behçet disease in Korea during three decades (1983-2012): chronological analysis of 3674 hospital-based patients. $\mathrm{Br} \mathrm{J}$ Dermatol 2014;170:458-61.

40 Kechida M, Salah S, Kahloun R, et al. Cardiac and vascular complications of Behçet disease in the Tunisian context: clinical characteristics and predictive factors. Adv Rheumatol 2018;58:32.

41 Hattori S, Kawana S. Behçet's syndrome associated with acute myocardial infarction. J Nippon Med Sch 2003;70:49-52.

42 Farouk H, Chilali KE, Said K, et al. Value of certain echocardiographic findings in the initial suspicion of Behçet's disease. Echocardiography 2014;31:924-30.

43 Moreira DM, da Silva RL, Vieira JL, et al. Role of vascular inflammation in coronary artery disease: potential of antiinflammatory drugs in the prevention of atherothrombosis. Inflammation and anti-inflammatory drugs in coronary artery disease. Am J Cardiovasc Drugs 2015;15:1-11.

44 Huet F, Akodad M, Fauconnier J, et al. Anti-inflammatory drugs as promising cardiovascular treatments. Expert Rev Cardiovasc Ther 2017; 15:109-25.

45 Ridker PM, Silvertown JD. Inflammation, C-reactive protein, and atherothrombosis. J Periodontol 2008;79:1544-51.
46 Ridker PM. Closing the loop on inflammation and atherothrombosis: why perform the CIRT and CANTOS trials? Trans Am Clin Climatol Assoc 2013;124:174-90

47 Ridker PM, Libby P, MacFadyen JG, et al. Modulation of the interleukin- 6 signalling pathway and incidence rates of atherosclerotic events and all-cause mortality: analyses from the canakinumab anti-inflammatory thrombosis outcomes study (CANTOS). Eur Heart J 2018;39:3499-507.

48 Ahn JK, Yu HG, Chung H, et al. Intraocular cytokine environment in active Behcet uveitis. Am J Ophthalmol 2006;142:429-34.

49 Park UC, Kim TW, Yu HG. Immunopathogenesis of ocular Behçet's disease. J Immunol Res 2014;2014:1-13.

50 Kim TW, Chung H, Yu HG. Chemokine expression of intraocular lymphocytes in patients with Behçet uveitis. Ophthalmic Res 2011;45:5-14.

51 Kim TW, Kang JS, Kong JM, et al. Maturation profiles of periphera blood dendritic cells in patients with endogenous uveitis. Immunol Lett 2012;142:14-19.

52 Alis D, Durmaz ESM, Civcik C, et al. Assessment of the common carotid artery wall stiffness by shear wave elastography in Behcet's disease. Med Ultrason 2018;20:446-52.

53 Emmi G, Silvestri E, Squatrito D, et al. Thrombosis in vasculitis: from pathogenesis to treatment. Thromb J 2015;13:15.

54 Owlia MB, Mehrpoor G. Behcet's disease: new concepts in cardiovascular involvements and future direction for treatment. ISRN Pharmacol 2012;2012:760484

55 Demirelli S, Degirmenci $\mathrm{H}$, Inci S, et al. Cardiac manifestations in Behcet's disease. Intractable Rare Dis Res 2015;4:70-5. 